DOI: $10.35643 /$ Info.25.1.2

Dossier temático: Miradas epistemológicas, históricas y conceptuales de las disciplinas de la información

\title{
Fundacionismo y ciencia de la información: una revisión crítica ${ }^{1}$ Foundationalism and information science: a critical review
}

\section{Ignacio Saraiva $^{\mathrm{a}}$}

\begin{abstract}
aAyudante del Departamento de Fuentes Documentales, Recursos y Servicios de Información del Instituto de Información (Facultad de Información y Comunicación, Universidad de la República, Uruguay). Correo electrónico: ignacio.saraiva@fic.edu.uy. ORCID: 0000-0002-7227927X
\end{abstract}

\section{Resumen}

En las diversas discusiones en torno a la cientificidad de la ciencia de la información, la postura de que es necesario clarificar e indagar acerca de los fundamentos del área, se posiciona como una de las más relevantes a la hora de comprender los problemas de la disciplina. Tal postura se sustenta bajo la idea de que las disciplinas científicas se definen por poseer un conjunto de fundamentos que las legitima. El objetivo del trabajo es exponer que la búsqueda de los fundamentos se encuentra enmarcada dentro del programa fundacionista. A su vez, se intenta presentar las críticas más importantes a este programa. Para ello, se plantean las principales características del fundacionismo. Luego, a partir de la obra de Rorty, se exponen los elementos de la filosofía epistemológicamente centrada, y se exhiben sus principales críticas. Se presentan los intentos más notorios a la hora de ofrecer una fundamentación para la ciencia de la información. A continuación, se defiende una postura no fundacionista para los problemas epistemológicos del campo. Se concluye que, desde una perspectiva no fundacionista, la importancia atribuida a la búsqueda de un fundamento no presenta relevancia sobre el estatus científico de la ciencia de la información.

Palabras clave: Epistemología de la ciencia de la información; Rorty; Fundacionismo; Anti fundacionismo; Filosofía de la ciencia. 


\begin{abstract}
In the various discussions around the scientific nature of information science, the position that it is necessary to clarify and inquire about the fundamentals of the area, is positioned as one of the most relevant when it comes to understanding the problems of the discipline. This position is support by the idea that the scientific disciplines are defined for having a set of foundations that legitimized them. The objective of this work is expose that the search of the foundations is framed within of the foundationalism program. In turn, an attempt is made to present the most important criticisms of this program. For this, the main characteristics of the foundationalism are raised. Then, from Rorty's work, the elements of epistemological centered philosophy are exposed, and his main criticisms are exhibited. The most notorious attempts to provide foundation for the information science are provided. Next, a non-foundationalist position is defenden for the epistemological problems of the field. It is concluded that, from a nonfoundationalist perspective, the importance assigned to the search for a foundation does not represent relevance on the scientific status of the information science.
\end{abstract}

Keywords: Epistemology of information science; Rorty; Foundationalism; Anti foundationalism; Philosophy of science.

Fecha de recibido: 24/04/2020

Fecha de aceptado: 11/05/2020

\title{
1. Introducción
}

La ciencia de la información, en tanto disciplina con pretensiones de cientificidad, ha llevado adelante diversos debates y discusiones sobre su estatus de conocimiento, así como de su potencialidad para ser considerada un conocimiento de tipo científico. Posicionar un campo como "ciencia" (ciencia de la información) supone un compromiso epistemológico muy fuerte. Al igual que el resto de las ciencias sociales, la ciencia de la información, a mediados del siglo $\mathrm{XX}$, intenta legitimar su estado cognitivo partiendo de una concepción de ciencia proveniente de las ciencias naturales. Ya comenzado el siglo XXI, muchas de estas discusiones siguen presentes y se instalan como imprescindibles a la hora de 
comprender el rol que debe jugar la disciplina en la actualidad. En buena parte de estas discusiones, se ven involucrados diversos problemas epistemológicos, así como también la clarificación de los límites disciplinares.

De igual forma, las discusiones y debates se han configurado en preguntas acerca de si las disciplinas que se ocupan de la información han resuelto su estatus científico, o interrogantes referidas a qué requisitos deben efectuar a la hora de consolidarse como una ciencia. Sobre esta base, tradicionalmente parece haberse considerado que esclarecer cuáles son los fundamentos de la ciencia de la información constituye una parte indispensable para ajustar y justificar su condición científica. Es así que, dentro de las discusiones, se acepta el supuesto de que los campos científicos detentan un conjunto de fundamentos que los define como tal, y que su búsqueda y elucidación permite estabilizar tanto los límites como esclarecer su nivel de cientificidad.

La presente investigación pretende analizar la noción de fundamentos disciplinares, así como delimitar bajo qué discusiones epistemológicas se han llevado adelante sus críticas y defensas. En este sentido, se pretende examinar críticamente la pertinencia de utilizar la idea de fundamentos dentro de la ciencia de la información. En virtud de ello, se advierten dos cuestiones; primeramente, parece aceptarse, implícitamente, la existencia de fundamentos del conocimiento; $\mathrm{y}$, en segundo lugar, se admite, explícitamente, la idea de que es lícito inspeccionar y clarificarlos, en orden de mejorar el estado cognitivo del campo.

Por lo cual, el objetivo del trabajo es exponer que la búsqueda de los fundamentos se encuentra enmarcada dentro de la tradición del programa fundacionista, y que existen buenas razones para criticar y abandonar este programa filosófico. A su vez, se considera que dilucidar los fundamentos de la ciencia de la información no repercute directamente en la mejora de las pretensiones de cientificidad de la disciplina.

La presunción de que la ciencia de la información requiere de fundamentos no se expone a discusión, lo que trae como consecuencia la asunción de algunas de las tesis y problemas del fundacionismo. Admitir la idea de fundamentos, conlleva posicionarse bajo un marco fundacionista, marco que ha sufrido diversas y fuertes críticas a partir de la segunda mitad del siglo XX. 
Para llevar adelante el análisis, en primer lugar, se presentan y exponen los principales argumentos planteados por el programa filosófico del fundacionismo. Esto permite comprender y contextualizar los debates en torno a la cientificidad de la ciencia de la información. En segundo lugar, se exhiben los aportes realizados por Richard Rorty en su obra La filosofía y el espejo de la naturaleza, y se debate a partir de las críticas realizadas a la filosofía epistemológicamente centrada como elemento clave para comprender las implicancias del fundacionismo. Luego, se examinan algunos de los intentos más relevantes dentro de la ciencia de la información a la hora de indagar y manifestar sus fundamentos. Por último, se defiende una perspectiva no fundacionista para comprender y analizar los problemas epistemológicos del campo. A su vez, se concluye que la importancia atribuida a la cuestión de los fundamentos es, en su mayoría, ilegítima. De igual forma, plantear los problemas epistemológicos de la ciencia de la información sobre la noción de fundamentos, parece no contribuir a una mejora del campo.

\section{Acerca del fundacionismo}

\subsection{De la justificación al edificio del conocimiento}

El fundacionismo es una de las corrientes epistemológicas más relevantes e influyentes, que ha dominado buena parte de los debates acerca de la forma en que se justifican nuestras creencias con respecto al mundo. El programa fundacionista se puede rastrear históricamente desde Platón, pasando Looke, Descartes y Kant. A su vez, suele incluirse al neopositivismo entre sus proyectos epistemológicos relacionados. Este punto no es menor, puesto que buena parte de las tesis centrales del programa serán atacadas y entrará en crisis en la segunda mitad del siglo XX (Di Gregori, 2006; Rorty, 1995, Sousa, 2012).

Tal corriente considera que existe algún tipo de creencias que no se encuentran soportadas por ningún otro elemento, a las cuales denomina básicas. Es decir, sustenta que estas creencias no necesitan otra fundamentación. Bajo estos términos, se puede decir que se trata de la base del resto del conocimiento y que intenta ofrecer una explicación de cómo se compone el mundo, aquellos que se encargan de dar un soporte. El fundacionismo suele asumir que las creencias básicas están exentas de cualquier duda, ya que son la mínima expresión del 
conocimiento legítimo. A su vez, son el punto de partida que permite acceder y derivar conocimiento legítimo. Tal vez la metáfora que mejor describe las pretensiones del fundacionismo, es la idea de que el conocimiento es una clase de edificio que encuentra su cimiento en los fundamentos básicos. No sólo la noción de que hay una base estable para el conocimiento toma sentido, sino que también adquiere importancia la idea de que la estabilidad propiciada faculta una acumulación, que concede como resultado conocimiento genuino. Al respecto, se puede estimar que:

Un fundacionista [...] sostiene que si tenemos creencias justificadas, no todas ellas están justificadas por otras creencias (es decir, que no todas ellas están justificadas inferencialmente), sino que tiene que haber un subconjunto de creencias justificadas que se justifican no inferencialmente. A las creencias cuya justificación no se basa en otras creencias - si es que las hay- se las conoce en epistemología como creencias "básicas"... (García, 2013, p. 25).

Es preciso subrayar dos características del programa fundacionista. Por un lado, la necesidad de poseer una base sólida, de la que no es posible dudar y con la cual se puede erigir nuevo conocimiento, y que refleja claramente la metáfora del edificio. Este punto implica la existencia de una base común externa, compartida por los individuos y que es presentada como una condición necesaria para las pretensiones del conocimiento. Por otro lado, habilita a suponer que, si el conocimiento se encuentra correctamente derivado de las creencias básicas, es legítimo.

Lo señalado hasta aquí, habilita a analizar otro punto importante para comprender las implicancias y los presupuesto del fundacionismo. Se puede dividir el total de creencias en dos grupos; por un lado, aquellas que son básicas, y, por otro lado, las que se encuentran justificadas. Las primeras no presentan una fundamentación para validarse, ya que representan los fundamentos últimos e infalibles con los que cuentan los individuos a la hora de ensamblar el edificio del conocimiento; a su vez funcionan como apoyo para el segundo tipo de creencias. Las segundas, que se encuentran sustentadas por las primeras, son aquellas que poseen un carácter no básico y se hallan derivadas del primer grupo, y por ello se puede decir que poseen una relación de dependencia. Esto posiciona al primer conjunto en una condición de independencia con respecto al resto del conocimiento. Se puede decir que hay una visión de la estructura del conocimiento, en donde existe una infraestructura y una superestructura. 
Tal punto permite vislumbrar algunos elementos del conocimiento que adquieren los individuos. El fundacionismo parece indicar que, si los individuos poseen conocimiento justificado, es porque poseen un conjunto de creencias básicas y otro no básico. Las creencias básicas que ostentan son el fundamento del conocimiento; mientras que, las creencias no básicas con las que se cuenta, en definitiva, se encuentran justificadas por alusión a los fundamentos.

Los intentos del fundacionismo por presentar una estructura del conocimiento lo más preestablecida posible no es un propósito librado al azar. Sino que es uno de los objetivos con los que intenta cumplir. La búsqueda y existencia de las creencias básicas o de un conjunto de fundamentos del conocimiento son elementos centrales del esquema propuesto. Lo que procura evitar con esto es una regresión infinita; es decir para el fundacionista la sucesión justificatoria de las creencias debe detenerse en determinado momento. A saber, la regresión de una justificación es suspendida por la existencia de los fundamentos básicos. ¿Qué quiere decir lo establecido anteriormente? Si un individuo intenta justificar una creencia, debe recurrir a otras creencias para poder fundamentarla, pero ¿qué acredita que las creencias utilizadas para justificar a otra estén fundamentadas? Si se sigue este punto, para el fundacionismo, se caería en una regresión infinita, en donde ninguna de las creencias posee algún tipo de justificación, en donde toda creencia se encuentra soportada por otra, que a su vez necesita la base de otra, lo que lleva a una cadena sinfín. A su vez, la disposición del argumento conlleva la posibilidad de caer en una circularidad, ya que sólo se obtendrían creencias justificadas en otras creencias, justificadas en otras creencias, etc. Para evitar estos puntos, es necesario contar con creencias básicas que admitan romper con la regresión y así contar con fundamentos que permitan avanzar en el conocimiento. Tal argumento se puede describir de la siguiente forma:

Cuando justificamos la creencia A, apelando las creencias B y C, todavía no hemos mostrado que A esté justificada. Sólo hemos mostrado que A está justificada si lo están B y C [...] la justificación de A está condicionada a la justificación de B y C. Pero, si toda justificación inferencial es condicional en este sentido, no hay nada de lo que pueda decirse que está justificada realmente, de un modo no condicional. Para cada creencia que intentamos justificar, siempre habrá una creencia ulterior de cuya justificación dependerá la justificación de la primera, $\mathrm{y}$, dado que éste es un regreso al infinito, no habría ninguna creencia que estuviera justificada más que de un modo condicional (Dancy, 2007, p. 74). 
En virtud de ello, si se acepta como válido el argumento de la regresión infinita, para el fundacionismo, se abandona la idea de saber cuándo el conocimiento se encuentra justificado, una consecuencia perjudicial para este programa. El fundacionismo encuentra la solución al problema bajo la noción de que el encadenamiento de justificaciones es interrumpida por la existencia de un conjunto de fundamentos, que es la base para el resto de las creencias (Dancy, 2007; Ketzer, 2011) ${ }^{2}$.

Los argumentos vertidos por el fundacionismo, en gran medida, presuponen la búsqueda de alguna estructura inmutable en el mundo, la existencia de elementos estáticos con la capacidad de fundamentar el conocimiento. En este presupuesto subyacen varios problemas fuertemente criticados, ya que supone que existe algún tipo de conocimiento dado a priori (Kalpokas, 2010; Sellars, 1971). De igual forma, aceptar la tesis del fundacionismo conduce a reparar en que los individuos pueden encontrar conocimiento dado y preestablecido, y que tal acontecimiento proporciona los fundamentos del conocimiento. Si esto es así, es porque se puede aseverar que hay un conjunto de conocimientos privilegiados sobre el mundo. Sumado a ello, se puede decir que hay un conjunto de creencias que son independiente a las capacidades cognoscitivas de los individuos, que se les impone ya que forman parte de la esencia de la realidad.

\subsection{De los fundamentos al espejo de la naturaleza}

Una vez señalados los principales rasgos del fundacionismo, es necesario mencionar otro de los sentidos y alcances que abarca. La noción de fundacionismo también se refiere a que las creencias y conocimientos se encuentran fundados en una base filosófica, y que la disciplina encargada de analizar e indagar sobre ellas, es la filosofía. Al respecto, Susan Haack considera que:

el «fundacionalismo» tiene también dos usos meta-epistemológicos: para referirse a la idea de que las pautas epistémicas tienen una base o fundamento objetivo; y para referirse a la idea de que la epistemología es una disciplina a priori cuya meta es legitimar o fundamentar nuestro supuesto conocimiento empírico... (Haack, 1997, p. 29).

La distinción realizada por Haack es significativa y abre otro campo de debate sustancial para la presente investigación ${ }^{3}$. Es sobre este punto que toma gran 
relevancia los aportes realizados por el filósofo estadounidense Richard Rorty, quien intenta atacar al programa fundacionista, y sobre todo la noción de que el conocimiento se encuentra fundamentado por la filosofía ${ }^{4}$.

Richard Rorty publica en 1976 una de sus obras más reconocidas, La filosofía y el espejo de la naturaleza (1995), en donde desarrolla una sólida crítica a la filosofía moderna, específicamente a los problemas mente-cuerpo, la concepción del conocimiento como representación exacta o la noción de fundamentos del conocimiento, y discute principalmente la imagen de la filosofía que se deriva de estos problemas, íntimamente relacionados con el programa fundacionista ${ }^{5}$. Rorty caracteriza tanto a estos problemas como a la visión filosófica obtenida como filosofía epistemológicamente centrada.

Considera que la filosofía epistemológicamente centrada es el resultado de algunos elementos vertidos mediante la filosofía moderna, y en particular a partir de los aportes recibidos en el siglo XVII. En este sentido, repara en que las contribuciones desarrolladas por Descartes, Locke y Kant terminan por constituir una imagen de la filosofía que dominará a la filosofía moderna, y que caracterizará de forma muy peculiar al conocimiento. Así pues, el presupuesto que dirige al conocimiento es que, la mente se halla diferenciada del cuerpo y que se encuentra facultada para analizar las representaciones que se presentan frente a ella. Las representaciones examinadas por la mente son la base del conocimiento, ya que capta de forma precisa los objetos que se presentan frente a ella. La victoria de tal idea da paso al surgimiento de varias metáforas oculares que se impondrán en el léxico de la filosofía moderno, a saber: el ojo de la mente que analiza las ideas o la mente como espejo que refleja la naturaleza. La imposición de estas metáforas dio como producto la suposición de que la filosofía es la disciplina específica encargada de examinar el conocimiento, y como resultado, ofrecer una adecuada exposición de la teoría del conocimiento.

En consecuencia, la tarea de indagar sobre el conocimiento es la tarea central para el quehacer filosófico. La filosofía y la teoría del conocimiento se colocan como los componentes encargados de dedicarse a pulir el espejo de la mente y de localizar los fundamentos. Sin el triunfo de esta concepción, las afirmaciones acerca de que la filosofía se dedica al "análisis conceptual”, las "explicaciones de 
los significados" o a la "lógica del lenguaje", no serían relevantes. Al respecto, Rorty repara en que:

los filósofos piensan que su disciplina se ocupa de problemas perennes [...] y se cristalizan en preguntas sobre los «fundamentos» del conocimiento. Descubrir estos fundamentos es descubrir algo sobre la mente, y al revés [...] la filosofía en cuanto disciplina se considera a sí misma como un intento de confirmar o desacreditar las pretensiones de conocimiento que se dan en la ciencia, en la moralidad, en el arte o en la religión [...] La filosofía puede tener carácter de fundamento en relación con el resto de la cultura, pues ésta es la acumulación de las pretensiones de conocimiento, y la filosofía debe juzgarlas. Puede hacerlo porque comprende los fundamentos del conocimiento, y encuentra estos fundamentos en un estudio del hombre-encuanto-ser-que-conoce [...] (Rorty, 1995, p. 13).

Lo aportado por Rorty permite vislumbrar que la metáfora cuasi visual de la mente como espejo que refleja la naturaleza, reparó en que la filosofía debe ocuparse de pulir y obtener imágenes claras acerca del mundo. Las representaciones mentales privilegiadas, reflejadas en el espejo, se transformarán en la base o fundamentos del conocimiento. Por consiguiente, la filosofía epistemológicamente centrada aboga que el conocimiento posee una base segura que lo sustenta. Este punto, admite realizar dos puntualizaciones; por un lado, que este programa filosófico se encuentra inscripto dentro del fundacionismo, en la medida de que intenta buscar algún tipo de sustento definitiva para el resto del conocimiento. Por otro lado, una vez que se emplaza a la filosofía como fundamento último del conocimiento, también se le otorga la capacidad de aportar un método con la cualidad de descubrir tal base.

El quehacer de la filosofía se centra en investigar y revelar los fundamentos del conocimiento, su esencia y analizar de qué manera el ojo de la mente comprende la naturaleza. La filosofía debe validar y legitimar las pretensiones de conocimiento (especialmente el científico) a través de las herramientas que posee. Acerca de este punto, Rorty consideraba que las metáforas oculares que dominaron la filosofía moderna terminan de posicionarla como el fundamento que sustenta el resto de las manifestaciones culturales y de las pretensiones del conocimiento. El método aportado por la filosofía es capaz de pulir el espejo de la mente de buena forma, para conocer adecuadamente la naturaleza. Rorty menciona que: 
La idea de que un armazón neutro y permanente cuya «estructura» puede mostrar la filosofía es la idea de que los objetos que van a ser confrontados por la mente, o las reglas que constriñen la investigación, son comunes a todo discurso, o al menos a todo discurso que verse sobre un tema determinado (Rorty, 1995, p. 288).

El programa de la filosofía epistemológicamente centrada se focalizó en la idea de que es necesario encontrar un conocimiento último acerca de la naturaleza, en hallar una estructura permanente y ahistórica que sirva de base para el desarrollo de los saberes. Asimismo, parece suponer que, si existen fundamentos y bases comunes del conocimiento, es porque es posible trasladar las diversas expresiones y disciplinas a un campo conmensurable, y encontrar un conjunto de reglas mínimas que sean comunes a todas ellas.

\section{La ciencia de la información y sus fundamentos}

\subsection{La búsqueda de una base común}

Hasta aquí se ha expuesto la noción de fundacionismo y las implicancias que posee para el conocimiento, y también se han expresado los principales elementos de la filosofía epistemológicamente centrada, programa que se reconoce plenamente como fundacionista. En adelante, se tratará de observar algunas de las relaciones que el programa fundacionista, y en particular algunas de las premisas de la filosofía epistemológicamente centrada, posee con las discusiones epistemológicas de la ciencia de la información.

Buena parte de las discusiones efectuadas en la ciencia de la información, legitiman las pretensiones de conocimiento sobre la idea de que posee un conjunto de fundamentos que le es propio y que es posible identificar. Se presupone que se posee un fundamento y que esclarecerlo es una tarea legítima y de gran relevancia disciplinar. Esto implica asumir implícitamente que existe algo como los fundamentos del conocimiento, y que son pasibles de investigar. A su vez, también compromete a considerar que la ciencia de la información posee fundamentos, y más específicamente, que las disciplinas científicas detentan fundamentos. Tales premisas se pueden encuadrar dentro de lo que Rorty catalogó como filosofía epistemológicamente centrada.

En virtud de ello, a la hora de justificar la cientificidad de la disciplina, se considera que es válido clarificar los fundamentos que definen a la ciencia de la 
información. Dilucidar sus pretensiones, implica demostrar que las prácticas del campo se respaldan en algún tipo de sustento filosófico. Los debates en torno a ello se presentan sobre la presunción de que es necesario contar con un sistema filosófico que oficie de soporte, que justifique y legitime el quehacer disciplinar (Amorim y Medeiros, 2017; Compton, 2015; Furner, 2010).

Se parte del supuesto de que ofrecer una visión acabada del fundamento de la disciplina es una tarea de gran relevancia a la hora de justificar las pretensiones de cientificidad. El trabajo de esclarecer los fundamentos es un trabajo legítimo y muy importante. Es así que, en parte de las discusiones epistemológicas de la ciencia de la información se encuentra la idea de que es imprescindible contar con una base filosófica que justifique sus pretensiones de cientificidad, y que se encargue respaldar el tipo de conocimiento que se produce. La filosofía se presenta como la encargada de clarificar y discernir el tipo de conocimiento elaborado por la disciplina.

Tal vez uno de los aportes que sintetiza de buena forma lo mencionado hasta el momento, son los realizados por Rendón Rojas. Rendón Rojas. En un intento de desmarcarse del anti-fundacionismo ha reparado en la necesidad de que la ciencia de la información cuente con fundamentos, legitimando el trabajo filosófico dentro del campo (Rendón Rojas, 2013a; 2014). Al respecto, aporta que:

Esta posición de no buscar un fundamento, recuerda uno de los relatos fantásticos del legendario barón Münchhausen quien contaba cómo después de haber caído en un pantano pudo salir de éste jalándose de los cabellos él mismo. Suena imposible porque no existe un punto de apoyo externo, pero es el mismo consejo que nos da un antifundacionismo exacerbado (Rendón Rojas, 2013a, p. 41).

Para Rendón Rojas, es necesario que la ciencia de la información, en tanto ciencia, cuente con un conjunto de fundamentos que le sirvan de base para su desarrollo. Da por sentado la existencia de fundamentos, ya que, si no fuera así, se caería en un sinsentido, en donde no se cuenta con ninguna base para el desarrollo cognitivo disciplinar. La metáfora planteada refleja claramente la idea del edificio del conocimiento (tan anhelada por el fundacionismo), en donde es indispensable contar con cimientos capaces de soportar el avance del conocimiento. Lo expuesto indica que si no hay fundamentos no hay punto de partida.

Las referencias a la necesidad de contar con un punto de apoyo externo, señalan lo manifestado por Rorty, en la medida de que se parte del supuesto de que existe 
una base común que es conmensurable y comparable a todos los campos. A su vez, es indispensable contar con fundamentos, pues si no fuera así, ¿qué frenaría una regresión infinita acerca de los elementos de la disciplina? Si no existe un punto de apoyo externo, se corre el riesgo de no lograr un avance sustantivo.

Rendón Rojas también ha impulsado la idea de que la ciencia de la información debe detentar un objeto de estudio común (2013b; 2013c). Al respecto, considera que se debe indagar acerca de un común denominador dentro de los diversos candidatos a objetos de estudio que existen, en orden de encontrar la mínima expresión que produzca consenso para la disciplina. Luego de analizar los múltiples aspirantes a objetos de estudio, concluye que el principio mínimo y común denominador es el informativo documental.

Si bien la búsqueda de un fundamento y la de un objeto de estudio parecen ejercicios distintos, ambos reflejan la aspiración de hallar una base sólida y común para legitimar el trabajo disciplinar. Este punto permite distinguir que se asume implícitamente la premisa de que existen fundamentos del conocimiento. Comprometerse con tal premisa conduce a admitir buena parte del programa fundacionista.

Es necesario remarcar que esta postura no solamente es sustentada por Rendón Rojas, sino que es una posición que habitualmente se encuentra dentro del área a la hora de esclarecer las pretensiones de cientificidad (Floridi, 2010; Hjorland, 2000; Mostafá, 2010). En virtud de ello, se puede aludir lo aportado por Ribeiro y Malheiro da Silva, quienes sustentan que:

[...] es importante considerar el vector de investigación, el cual es particularmente relevante en el paradigma científico informacional y está siempre alineado con las bases teóricas y el enfoque metodológico [...] La existencia de un soporte teórico-metodológico, es en sí mismo, una diferencia esencial con el paradigma tradicional en donde la teoría y el método estaban ausentes y/o se confundían con actividades técnicas. Desde una perspectiva científica, la investigación ya no es sinónimo de describir utilizando estándares aplicados sin criterio y pasa a significar saber, analizar, interpretar y explicar la realidad informacional que es el objeto de estudio... (Ribeiro y Malheiro da Silva, 2016, p. 122, traducido).

Los autores aluden a que la existencia de una base teórica es la que permite el desarrollo científico, lo que concede una dirección a la investigación del campo. La base teórica es el sustrato que le da sentido al quehacer disciplinar, es lo que diferencia y consolida a una ciencia de cualquier otro saber. Tal punto indica que 
la transición de una actividad a una ciencia está marcada por la adquisición de fundamentos. Más aún, la base teórica adquirida posibilita la comprensión y delimitación del objeto de estudio.

En cuanto a lo referido anteriormente, se considera que, al examinar los problemas epistemológicos de la ciencia de la información, se concede de forma implícita la existencia de fundamentos, y que sea legítimo e indispensable investigar y examinarlos. Parece no someterse a debate las premisas, implicancias y consecuencias acerca del fundacionismo.

\subsection{De la base común a la filosofía}

Ya en la década de los 80' del siglo XX, Bertram Brookes publica una serie de artículos sobre los fundamentos de la ciencia de la información, en donde emprende la tarea de reconstruir varios elementos de la disciplina en orden de explicar su dinámica ${ }^{6}$. Cabe resaltar que, en el primero de sus artículos, se centra en los aspectos filosóficos. Este elemento no es menor, ya al tratar los fundamentos disciplinares considera que es imprescindible clarificar su base filosófica, asumiendo que es la disciplina que sirve de fundamento para el resto de las pretensiones del conocimiento. Una lectura rortyana de este hecho, indica que no es casual que el estudio realizado comience por los aspectos filosóficos, sino que es producto de la visión en donde la filosofía es el sustento del resto de las manifestaciones culturales. La filosofía es el último lugar en donde se puede discutir sobre cómo está compuesto el mundo.

Al estudiar los aspectos filosóficos Brookes (1980a) repara en que una forma de delimitar las fronteras de la disciplina es proclamar como propio el mundo del conocimiento objetivo o tercer mundo establecido por Popper, ya que ninguna otra disciplina lo ha designado como suyo. Es en este espacio donde la ciencia de la información encuentra lo que estudia, un campo que le otorga autonomía e identidad.

Para Brookes, las disciplinas construyen estructuras teóricas que permiten su crecimiento, y las cuales deben desarrollarse. En virtud de ello, hay dos formas de producir su crecimiento. Por un lado, se puede aportar a la superestructura que encuentra sus bases en la teoría. Mientras que, por otro lado, se puede hacer crecer a la disciplina fortaleciendo y extendiendo sus bases teóricas. Para ambas tareas es 
necesario contar con fundamentos o supuestos básicos lo más clarificados posibles. Brooks considera que:

La estructura teórica de una ciencia nunca está completa [...] Todos los aspectos permanecen abiertos, ofreciendo nuevos problemas. Una vez que el núcleo de una nueva teoría es visiblemente coherente, hay dos formas en que puede desarrollarse: primero, mediante el crecimiento de la superestructura que descansa sobre los cimientos iniciales, extendiendo así el rango de la teoría; y, en segundo lugar, profundizando o fortaleciendo sus cimientos (Brookes, 1980a, p. 125, traducido).

En ambos casos se observa que el desarrollo disciplinar está directamente relacionado a la capacidad que posean sus bases filosóficas de sustentar o de expandirse. Si se puede ofrecer una versión de los fundamentos de la ciencia de la información se aportará a su desarrollo. Es necesario direccionar las discusiones del área hacia la filosofía, ya que una clarificación en ese ámbito es indispensable. Tal punto también puede observarse en abordajes más contemporáneos. Cornelius (2014) al referirse al lugar que debe ocupar la información y la teoría de la información en la ciencia de la información, centrales a la hora de ofrecer una mejor reconstrucción de la disciplina y de su estatus, considera que:

...parecería obvio, útil y necesario tener una teoría de la información que pueda ser usada como base para la construcción de una ciencia de la información. De hecho, parece anómalo hacer afirmaciones sobre una ciencia de la información a menos que sepamos el lugar de la información dentro de ella... Necesitamos saber qué podemos hacer con una definición de información o una teoría de la información. También necesitamos saber cómo nos encontramos en relación con otras disciplinas, incluso si tenemos una teoría o definición de información satisfactoria. Tenemos que demostrar que la teoría del conocimiento que subyace a nuestras acciones es suficiente para sostener la pretensión de ser una disciplina digna de reconocimiento como tal por otros campos. Para ello, necesitamos demostrar que tenemos las características de un campo académico de estudio. (Cornelius, 2014, p. 184, traducido).

Cornelius repara en que si el área pretende consolidar sus pretensiones de cientificidad debe estabilizar una base de fundamentos para edificarse como ciencia. Es necesario poseer una teoría de la información que sustente y direccione el trabajo, que determine qué sitio debe ocupar la información, así como establecer una definición de información que dé cuenta de la labor de la ciencia de la información. Esto va a posibilitar determinar los límites disciplinares y las diferencias con el resto de los campos, puesto que las diferencias disciplinares vienen dadas por el conjunto de fundamentos que cada una posee. 
Si la ciencia de la información procura dilucidar su estatus debe demostrar que posee una base teórica amplia que da sustento a sus prácticas. Para Cornelius hay características que definen a las ciencias, por lo que la ciencia de la información debe ajustar sus rasgos a estas características. Indica que las disciplinas que ostentan características de ciencias, lo han logrado ya que han clarificado adecuadamente sus fundamentos.

Desde otro punto de vista, Wilson (2003), al examinar la naturaleza de la investigación de la ciencia de la información, considera que el campo adolece de cohesión ya que no trabaja con un objeto de estudio único. La información, su objeto de estudio, se presenta en varios niveles puesto que no se trata de un concepto unitario, lo que permite la construcción de diversas teorías. Repara en que no puede existir un concepto que unifique el campo, ya que la información admite diversos enfoques. De acuerdo con esto, es necesario contar con un marco filosófico que le permita al investigador guiar su elección sobre qué método utilizar a la hora de examinar el nivel del objeto con el que trabaja, y menciona que:

...el método sin un marco filosófico que determine por qué se emplea un método particular y qué visión de la realidad sostiene el investigador es puramente mecanicista... si deseamos comprender el mundo de los usuarios de la información ... necesitamos herramientas conceptuales que hayan sido diseñadas para fomentar esa comprensión. Sugiero que la fenomenología ofrece esas herramientas conceptuales, ya sea que las derivemos del trabajo en sociología, psicología, educación o cualquier otra disciplina... (Wilson, 2003, p. 447-450, traducido).

Para Wilson los rasgos que definen a una ciencia es la utilización de un método. Buena parte de la incorporación y utilización de un método está directamente asociada a la elección de un sistema filosófico que lo respalde. Si lo que garantiza la cientificidad de una disciplina es su método, la filosofía brinda sus fundamentos, puesto que es imprescindible contar con herramientas conceptuales que den soporte a las decisiones metodológicas y disciplinares. Por lo que concede que sea ineludible escoger un marco filosófico. La filosofía puede aportar este tipo de herramientas, ya que es la encargada de fundamentar, analizar y direccionar el conocimiento.

En virtud de ello, considera que el mejor marco filosófico para la ciencia de la información es la fenomenología. La fenomenología ha demostrado aportar 
instrumentos capaces de encauzar el trabajo disciplinar, de manera que si se logra articular como fundamento del área se logrará a avanzar como ciencia. Aquí lo que se intenta realizar es legitimar la actividad disciplinar, al exponer que se adecua a las condiciones que impone un marco filosófico. Se inserta la discusión en un plano donde los problemas se resuelven en la medida que se opte por un fundamento y no como un resultado de la dinámica de la disciplina.

Esta forma de justificar el conocimiento apunta a que las pretensiones de cientificidad se resuelven con un elemento externo a su funcionamiento y que la resolución no surge desde dentro de un campo, sino que proviene de la filosofía. Una disciplina se considera ciencia no por elementos internos de su funcionamiento sino por elementos externos que le aportan una base para su desempeño. Cabe recordar que los planteos realizados por Rendón Rojas se dirigen en la misma dirección. Wilson agrega que:

Hay una razón adicional para adoptar un enfoque filosófico claro y coherente para nuestra comprensión del mundo y nuestros intentos de entenderlo. Es decir, entonces podemos fundamentar firmemente nuestros intentos de educación de investigación en ese marco filosófico y transmitir a los estudiantes la comprensión de que el método por sí solo no es suficiente para un programa de investigación, nosotros (y ellos) necesitamos un lugar firme en el que apoyarnos... (Wilson, 2003, p. 451, traducido).

Wilson señala que se debe contar con una base en la cual respaldar sus decisiones metodológicas, ya que esto permitirá una mejor comprensión del mundo. La filosofía se presenta como la disciplina que debe guiar al resto de las actividades del conocimiento por un camino seguro. No sólo el método se posiciona como criterio de cientificidad, sino que la noción de fundamentos, como base para la disciplina, se sitúa como elemento de gran relevancia.

Más recientemente, Gnoli (2019) analiza algunas nociones básicas de la ciencia de la información a partir de la teoría de los niveles de la realidad. Concluye que los fundamentos deben localizarse en la idea de mentefactos y sus diversos niveles, y no en la filosofía de la información proporcionada por Floridi. Considera que:

Una consecuencia de esto parece ser que, si bien la filosofía de Floridi puede ser relevante para la bibliotecología y ciencia de la información, también puede serlo para otras disciplinas que se ocupan de la información en diferentes niveles [...] la filosofía de la información en el sentido básico no parece tener ninguna relación exclusiva con la bibliotecología y ciencia de la información. Una base más específica para la bibliotecología y ciencia de la información debería entonces ser investigada en su propio nivel particular, el 
nivel de los hechos mentales. De lo contrario, existe el peligro de que otros fuera de la disciplina continúen siendo los principales contribuyentes (Gnoli, 2019, párr. 44, traducido).

Para Gnoli, la filosofía de la información no es la mejor opción como fundamento ya que puede ser utilizada por diversas disciplinas, y no presenta una visión específica de los fenómenos estudiados en el campo. Es necesario que la ciencia de la información cuente con una base filosófica específica para comprender los hechos a los que se ocupa y discernir el nivel de la realidad que le es propio. Esto es indispensable, en la medida que los fundamentos le van a permitir diferenciarse del resto de los saberes.

La discusión presentada por Gnoli es representativa del abordaje que se lleva adelante a la hora de discutir sobre los fundamentos de la ciencia de la información. Tales discusiones suelen partir de la noción de que una disciplina requiere de fundamentos, y que la idea de fundamentos es válida. Esto lleva a asumir buena parte del programa fundacionista, en la medida de que no se discute sobre la existencia de fundamentos. Situar la discusión acerca de los fundamentos en el contexto de una disputa entre marcos filosóficos, lleva a considerar que la cientificidad del campo se resolverá mediante una decisión externa a la dinámica interna de la disciplina, y que depende de una dilucidación filosófica.

Estas discusiones parecen indicar que, la ciencia de la información consolidará sus fundamentos en la medida que pueda adecuar su funcionamiento a un marco filosófico determinado, sea la filosofía de la información, la epistemología social, la teoría de los niveles de la realidad, la hermenéutica, la fenomenología o cualquier otro sistema filosófico de turno.

\section{Una perspectiva no fundacionista}

\subsection{De Rorty a la ciencia de la información: un diálogo posible}

Lo analizado hasta aquí permite señalar que, si bien dentro de la ciencia de la información no se encuentra una defensa explícita de todos los elementos del programa fundacionista ni de la filosofía epistemológicamente centrada, se encuentran puntos de contacto entre los planteamientos. Se pueden localizar elementos característicos, como por ejemplo es la idea de que las disciplinas se sustentan por una base o conjunto de fundamentos filosóficos. También se 
encuentra presente la noción de que el trabajo filosófico consiste en proporcionar y analizar los fundamentos.

Las preguntas sobre los fundamentos del conocimiento toman relevancia ya que esclarecerlos se vuelve una tarea central. Este tipo de ejercicios supone que la cientificidad de la ciencia de la información depende de una elucidación filosófica sobre sus bases, es así que sus premisas básicas se encuentran enquistadas en algún sistema filosófico.

Sobre este presupuesto, se presume que la filosofía es el ámbito en donde el conocimiento debe comparecer y demostrar que encuentra una buena relación con la naturaleza. Rorty (1995), al analizar estos elementos, considera que son parte del programa que asume que el conocimiento es un ejercicio de confrontar las representaciones mentales con los objetos del mundo, y que sitúa a la filosofía como la disciplina con los elementos necesarios para evaluar esta relación ya que es el último recinto en el cual se puede reducir el conocimiento. Reparar en que las disciplinas requieren de una base filosófica es producto de instalar a la filosofía como el sustento del resto de las manifestaciones culturales. Desde una perspectiva rortyana, el resultado de asumir las metáforas oculares sobre dinámica y naturaleza del conocimiento es emplazar a la filosofía al lugar de descubrir algún tipo de estructura que sustenta el mundo. Es pertinente recordar algunos puntos que Rorty señaló sobre la noción de la filosofía como disciplina base para el resto de las acciones de conocimiento. Apuntaba que:

la idea de la filosofía en cuanto disciplina fundamental que «sirve de base» a las pretensiones de conocimiento se vio consolidada... Era el área de la cultura donde se tocaba fondo, donde se encontraban el vocabulario y las convicciones que permitían explicar y justificar las actividades propias en cuanto intelectual, y descubrir, por tanto, el significado de la propia vida (Rorty, 1995, p. 14).

Por lo que sí se emplaza a la filosofía a la base del conocimiento es porque la actividad filosófica es la encargada de sostener a las pretensiones del conocimiento, así como de analizarlas y direccionar sus decisiones. En este sentido, cuando la ciencia de la información considera que posee fundamentos, asume las premisas de que la filosofía debe justificar y juzgar su conocimiento.

Cabe señalar que cuando Brookes (1980a) se preocupa por los fundamentos filosóficos que establecen los límites del campo, admite que la filosofía juega un papel preponderante a la hora de esclarecer sus pretensiones de conocimiento. En 
consecuencia, el progreso disciplinar parece estar sujeto a la posibilidad de que se aumenten la base de fundamentos. Hay un desplazamiento desde la dinámica disciplinar hacia una dinámica filosófica, en donde el avance de la disciplina pasa por esclarecer supuestos filosóficos.

Lo planteado por Wilson (2003), va un paso más adelante y asume que la disciplina necesita una base filosófica que logre encauzar la investigación. Indica que es pertinente seleccionar un sistema filosófico que le de soporte y que se adecue a su funcionamiento. Esta visión sustenta que esclarecer el estatus de la disciplina pasa primero por introducir un modelo filosófico para dilucidar sus problemas. La filosofía asume un papel normativo para la disciplina, puesto que brindar una adecuación correcta del campo a las características del sistema filosófico.

De acuerdo con esto, la discusión sobre los fundamentos de la ciencia de la información parece articularse en dos premisas que direccionan la discusión:

a) la ciencia de la información, en tanto disciplina con pretensiones de conocimiento, debe esclarecer sus fundamentos mediante un ejercicio filosófico.

b) Es lícito y necesario optar por un sistema filosófico de turno que sustente los fundamentos, en orden de lograr un progreso para la disciplina.

En virtud de ello, colocar los problemas epistemológicos de la ciencia de la información en un plano de dilucidación filosófica, se convierte en una tarea necesaria. Se puede considerar que tanto puede aportar una argumentación filosófica a priori sobre el funcionamiento de una disciplina, y cabe cuestionar si una discusión situada en el plano filosófico aporta al desempeño del campo. Las preguntas sobre cuáles son los fundamentos de la ciencia de la información, o sobre si la ciencia de la información posee fundamentos se tornan centrales a la hora de especificar bajo qué condiciones el campo es científico.

Así pues, es factible determinar que esta estrategia se identifica como parte del programa fundacionista y propio de la filosofía epistemológicamente centrada. Desde una perspectiva rortyana no es posible determinar los fundamentos del conocimiento, ya que no existe algo así como los fundamentos. A su vez, no se puede determinar un conjunto de estructuras estáticas que componen el mundo, ni tampoco es viable ofrecer una imagen de los fundamentos que sustentan el conocimiento. 


\section{2. ¿Ciencia de la información sin fundamentos?}

Si bien la postura defendida hasta este momento no es mayoritaria dentro de la ciencia de la información, recientemente Buschman (2017), en orden de diagnosticar y atacar los problemas epistemológicos, considera que es necesario superar la visión de la epistemología como búsqueda de un fundamento, por lo que es imprescindible ofrecer un panorama capaz de articular las contribuciones de la disciplina, para lo cual realiza un análisis a partir de Dewey.

Cabe recordar que para Rorty, Dewey (junto con Wittgenstein y Heidegger) fue de los primeros filósofos en sospechar que la epistemología se encontraba mal dirigida y que había que emanciparse de la concepción representacionista y de la búsqueda de fundamentos. Parte de la obra de Dewey busca ser más terapéutica y edificante y, en consecuencia, abandonar la visión de la filosofía en cuanto disciplina básica del conocimiento. Para Buschman (2017) este punto no es menor, ya que es necesario reconfigurar los debates filosóficos de la ciencia de la información, en orden de renunciar a la búsqueda de una base filosófica común para la disciplina. En consecuencia, observa que:

los problemas de LIS con la epistemología provienen de diversas fuentes: la misma epistemología, la combinación de la bibliotecología con la ciencia de la información y la búsqueda de una base común de las profesiones de la información, sus herramientas y sus instituciones. Tal fundamento teórico no es posible... (Buschman, 2017, p. 210, traducido).

Buschman, en la misma línea que Rorty, aclara que las pretensiones de cientificidad de la ciencia de la información no se resolverán mediante la búsqueda de una base filosófica, ya que esto es imposible puesto que no existe tal fundamento. A su vez, la visión que domina buena parte de los debates epistemológicos, responden a la idea de que existe un fundamento común para el conocimiento.

En este sentido, Zwaldo (1997) al discutir sobre la existencia de una filosofía para la bibliotecología y ciencia de la información, considera que no es necesario contar con una base filosófica que direccione a la disciplina. La bibliotecología, en orden de convertirse en una ciencia, ha intentado demostrar cuál es la mejor filosofía para su fundamento. El único resultado obtenido es la confusión para los practicantes de la disciplina. Zwaldo reafirma los argumentos de Rorty y concluye que: 
Las sugerencias de Rorty para curar los delirios de los filósofos se aplican a los delirios similares de los bibliotecarios. Los bibliotecarios deben usar métodos que funcionen, que sirvan a los fines de la biblioteca, sus usuarios y la comunidad, en lugar de tratar de justificar afirmaciones privilegiadas de la verdad... La falta de un "algoritmo de elección" para decirnos qué teoría representa con precisión la realidad objetiva ha afectado la física y la química, la biología y la psicología, tanto como la bibliotecología (Zwaldo, 1997, p. 119, traducido).

Zwaldo repara en que las pretensiones de cientificidad de la bibliotecología y ciencia de la información remiten antes a la capacidad de sistematizar y resolver problemas genuinamente disciplinares y no a la adopción de algún sistema filosófico o a la clarificación de sus fundamentos. Indica que, gran parte de las discusiones disciplinares, se centran en encontrar algún tipo de elemento externo a su funcionamiento, mientras que se deja de lado la capacidad de estructurar sus logros internos.

Se puede ultimar que las pretensiones de cientificidad de la ciencia de la información no se consolidarán a través de algún esclarecimiento de sus bases filosóficas o una mejora en la articulación de fundamentos. Las preguntas en torno a estos elementos no representan una mayor relevancia a la hora de mejorar su estado cognitivo. Ni la pregunta ni la respuesta acerca de sus fundamentos repercuten directamente en el estatus científico del área. Situar a la filosofía como la encargada de evaluar la actividad del conocimiento representa al programa de la filosofía epistemológicamente centrada.

Sobre esta base, se entiende que la idea de que la ciencia de la información posea un fundamento se encuentra fuertemente asociada a la certidumbre de que dar con un sistema filosófico que articule su labor, es constituir buena parte del estatus disciplinar. Para Rorty, estos elementos son propios de la tradición conllevan a un deseo de constricción, un deseo de encontrar un conjunto de reglas comunes a todas las investigaciones con la capacidad de dirigir y explicar el conocimiento.

\section{Conclusiones}

A partir de lo expuesto, se puede considerar que dentro de la ciencia de la información se acepta implícitamente la estrategia de indagar y justificar los fundamentos de la disciplina, en orden de dilucidar sus pretensiones de cientificidad, sin cuestionar qué derivaciones y qué problemas epistemológicos acarrea tales ejercicios. Se da por sentado que tiene algún sentido examinar sobre 
los fundamentos. Por lo que, en estos debates se presupone un marco de fundacionista.

Este punto se puede resumir de la siguiente manera: en el área subyace el supuesto de que es necesario ofrecer un conjunto de fundamentos, ya que es un rasgo de cientificidad de las disciplinas consolidadas. Así, se instala la noción de que es imprescindible definir y presentar los fundamentos.

La creencia de que las pretensiones de conocimiento de una disciplina se encuentran justificadas en cuanto poseen fundamentos, y que el trabajo filosófico es el encargado de dar con esta base, deviene del programa fundacionista. A su vez, la metáfora del edificio del conocimiento está presente, puesto que se parte del supuesto de que se debe poseer una base que le sirva de cimiento.

Parte de la ciencia de la información asume que la filosofía juega un papel preponderante a la hora de esclarecer la cientificidad del campo. Se coloca a la filosofía como la encargada de hallar y ofrecer una imagen clara de los fundamentos de la disciplina. Es así que, se presume que la cientificidad se dirime en tanto se pueda presentar una representación consistente de sus fundamentos. En este sentido, se considera que en los debates epistemológicos subyace el programa de la filosofía epistemológicamente centrada. Se repara en que una mejora cognitiva del campo se puede derivar de una fundamentación filosófica. Se supone que una justificación filosófica aportará mayor cientificidad a la disciplina. Esta premisa es introducida de forma implícita y sustenta que el conocimiento necesita algún tipo de fundamentación. En esta labor, se le asigna a la filosofía la capacidad de ser la base del conocimiento, de fundamento último de la actividad del conocimiento. Esto lleva a considerar que los problemas epistemológicos de la ciencia de la información encuentran respuesta en una dilucidación filosófica.

Desde una perspectiva rortyana, se puede meditar que la filosofía es presentada de dos formas distintas. La primera, implica un tono descriptivo y repara en que la filosofía es la encargada de descubrir los fundamentos. Y la segunda, supone un tono normativo, en donde se le asigna a la filosofía la capacidad de juzgar cuál es el mejor sistema filosófica para la disciplina.

Al respecto, se puede concluir que la trascendencia adjudicada a las discusiones sobre los fundamentos, desde una perspectiva rortyana y no fundacionista, son 
ilegítimas y no repercuten directamente en la mejora de las pretensiones científicas de la disciplina.

\section{Referencias bibliográficas}

Alston, W. P. (1976). Two types of foundationalism. The Journal of Philosophy, 73(7).

Amorim, I. S., y Medeiros, M. B. B. (2017). Apropriação da filosofia de Deleuze na Ciência da informação: Um estudo quantitativo. InCID: Revista de Ciência da Informação e Documentação, 8(2). Recuperado de https://doi.org/10.11606/issn.2178-2075.v8i2p69-91

Brookes, B. C. (1980a). The foundations of information science. Part I. Philosophical aspects. Journal of Information Science, 2(3-4), 125-133. Recuperado de https://doi.org/10.1177/016555158000200302

Brookes, B. C. (1980b). The foundations of information science: Part II. Quantitative aspects: classes of things and the challenge of human individuality. Journal of Information Science, 2(5), 209-221. Recuperado de https://doi.org/10.1177/016555158000200502

Brookes, B. C. (1980c). The foundations of information science: Part III. Quantitative aspects: objective maps and subjective landscapes. Journal of Information Science, 2(6), 269-275. Recuperado de https://doi.org/10.1177/016555158000200602

Brookes, B. C. (1981). The foundations of information science: Part IV. Information science: the changing paradigm. Journal of Information Science, 3(1), 3-12. Recuperado de https://doi.org/10.1177/016555158100300102

Buschman, J. (2017). Once more unto the breach: "Overcoming epistemology" and librarianship's de facto Deweyan Pragmatism. Journal of Documentation, 73(2), 210-223. Recuperado de https://doi.org/10.1108/JD-04-2016-0052 
Compton, B. W. (2015). Parallax ontology and the philosophy of information. Library Trends, 63(3), 555-573. Recuperado de https://doi.org/10.1353/lib.2015.0001

Cornelius, I. (2014). Epistemological challenges for information science: constructing information. En F. Ibekwe y T. M. Dousa (Eds.), Theories of Information, Communication and Knowledge: A Multidisciplinary Approach (pp. 181-203). Recuperado de https://doi.org/10.1007/978-94007-6973-1_8

Dancy, J. (2007). Introducción a la epistemología contemporánea. España: Tecnos.

Di Gregori, M. C. (2006). La fundamentación racional del conocimiento: programas fundamentistas. En Olivé, L. (comp.) Racionalidad epistémica. España: Trotta; Consejo Superior de Investigaciones Científicas (Enciclopedia Iberoamericana de filosofía).

Fernández Membrive, M. (2015). Frente al imposible espejo de la naturaleza: Rorty y Habermas. Daimon, 64. Recuperado de https://doi.org/10.6018/daimon/168381

Floridi, L. (2010). Biblioteconomia e Ciência da Informação (Bci) como filosofia da informação aplicada: uma reavaliação. InCID: Revista de Ciência da Informação e Documentação, 1(2), 37. Recuperado de https://doi.org/10.11606/issn.2178-2075.v1i2p

Furner, J. (2010). Philosophy and information studies. Annual Review of Information Science and Technology, 44(1), 159-200. Recuperado de https://doi.org/10.1002/aris.2010.1440440111

García, C. L. (2013). Introducción. En García, C. L., Eraña, A., y King Dávalos, P. (comp.). Teorías contemporáneas de la justificación epistémica. UNAM, Instituto de investigaciones filosóficas.

Gnoli, C. (2019). Levels of information and library and information science as a science of mentefacts. Proceedings of CoLIS, the Tenth International 
Conference on Conceptions of Library and Information Science.

Information Research, 24(4). Recuperado de http://InformationR.net/ir/244/colis/colis 1903.html

Haack, S. (1997). Evidencia e investigación: Hacia la reconstrucción en epistemología. España: Tecnos.

Hjorland, B. (2000). Library and information science: Practice, theory, and philosophical basis. Information Processing \& Management, 36(3). Recuperado de https://doi.org/10.1016/S0306-4573(99)00038-2

Iannaccone, L. (1999). Regreso justificatorio y fundacionalismo. En Sota, E. y Urtubey, L. (ed.). (1999). Epistemología e historia de la ciencia. Selección de trabajos de las IX Jornadas, 5(5). Recuperado de https://rdu.unc.edu.ar/handle/11086/3398

Kalpokas, D. (2005). Richard Rorty y la superación pragmatista de la epistemología. Buenos Aires: Ediciones del Signo.

Kalpokas, D. (2010). Dewey y el mito de lo dado. ENDOXA, 1(26). Recuperado de https://doi.org/10.5944/endoxa.26.2010.30

Ketzer, P. (2011). Fundacionalismo: Da tradição às teorias moderadas. Argumentos - Revista de Filosofia, 5. Recuperado de http://www.periodicos.ufc.br/argumentos/article/view/18990

Mostafá, S. P. (2010). Epistemología ou filosofía da ciencia da informacao? Inf. \& Soc.:Est., 20(3). Recuperado de https://bdpi.usp.br/bitstream/handle/BDPI/14982/art_MOSTAFA_EPISTE MOLOGY_OR_PHILOSOPHY_OF_INFORMATION_SCIENCE_2010. pdf? sequence $=1$

Navia, R. (2008). Richard Rorty: emplazamiento a la tradición filosófica. Montevideo: Departamento de Publicaciones de la Facultad de Humanidades y Ciencias de la Educación, Universidad de la República. 
Rendón Rojas, M. Á. (2013a). Una epistemología dialéctica de la Ciencia de la Información. Entre Ariadna y Penélope. En VI Encontro Ibérico EDICIC. Recuperado de http://eprints.rclis.org/22855/

Rendón Rojas, M. Á. (2013b). Introducción. En Rendón Rojas, M. Á. (comp.), El objeto de estudio de la bibliotecología/ documentación/ciencia de la información: propuestas, discusión, análisis y elementos comunes, VIIXIII. México: UNAM, Instituto de Investigaciones Bibliotecológicas y de la Información.

Rendón Rojas, M. Á. (2013c). Reflexiones finales. En Rendón Rojas, M. Á. (comp.), El objeto de estudio de la bibliotecología/ documentación/ciencia de la información: propuestas, discusión, análisis y elementos comunes, 275- 295. México: UNAM, Instituto de Investigaciones Bibliotecológicas y de la Información.

Rendón Rojas, M. A. (2014). El objeto de estudio en Biblioteconomía. Un problema con implicaciones lingüísticas, pragmáticas y epistemológicas. En Orozco Tenorio, J. M., Cobos Flores, A. y Ruiz Figueroa, R. (comp.), Foro Nacional de Profesionales de la Información. México: ENBA.

Ribeiro, F. y Malheiro da Silva, A. (2016). The Epistemological Maturity of Information Science and the Debate around Paradigms. En M. Kelly y J. Bielby (eds.), Information cultures in the digital age: A Festschrift in Honor of Rafael Capurro. Wiesbaden: Springer.

Rorty, R. (1995). La filosofía y el espejo de la naturaleza. Madrid: Cátedra.

Saraiva, I. (2019). La ciencia de la información y su objeto de estudio: una perspectiva crítica a partir de Richard Rorty. Cuadernos De FilosofíA, (37), 29-52. Recuperado de http://revistasacademicas.udec.cl/index.php/cuadernos_de_filosofia/article /view/1336

Sellars, W. (1971). El empirismo y la filosofía de lo mental. En Sellars, W. Ciencia, percepción y realidad. Madrid: Tecnos. 
Sousa, C. J. de (2012). O problema da crenca e da justificacao nas epistemologias naturalizadas de David Hume e Alvin Goldman. Revista electrónica: Prodocencia, 1(1). Recuperado de http://www.uel.br/revistas/prodocenciafope/pages/arquivos/NOVOS\%20T EXTOS\%2006\%20a\%2014/CLAUDINEY\%20SOUSA\%20-

\section{\%20FILOSOFIA.pdf}

Van Fraassen, B. C. (1992). Después del fundacionismo: Entre el círculo vicioso y el regreso al infinito. Revista de Filosofía DIÁNOIA, 38(38). Recuperado de

http://dianoia.filosoficas.unam.mx/index.php/dianoia/article/view/595/600

Wilson, T. D. (2003). Philosophical foundations and research relevance: issues for information research. Journal of Information Science, 29(6), 445-452. Recuperado de https://doi.org/10.1177/0165551503296002

Zwadlo, J. (1997). We don't need a philosophy of library and information science: We're confused enough already. The Library Quarterly, 67(2), 103-121. Recuperado de https://doi.org/10.1086/629928

\section{Notas}

${ }^{1}$ El presente artículo recoge parte de la tesis de maestría titulada "El objeto de estudio de la ciencia de la información: un análisis epistemológico", defendida en 2019.

${ }^{2}$ Este punto es uno de los más discutidos y criticados. Algunos de los debates se centran bajo las preguntas de cómo se determina las creencias básicas, o cómo están compuestas, sean empíricas, teóricas o lógicas. En la literatura también se pueden encontrar diversas posturas y discusiones sobre el regreso justificatorio. Para estos debates, se puede consultar: García (2013); Haack (1997); Iannaccone (1999). Aquí no se ha decidido no profundizar en estos, ya que el objetivo del apartado es presentar las principales características del fundacionismo.

${ }^{3}$ La distinción realizada Haack se ubica en las discusión y críticas al fundacionismo, para ver otros análisis sobre el mismo punto se puede consultar: Altson (1976); Sellars (1971); Van Frassen (1992). 
4 Recientemente, se ha ofrecido una reconstrucción de algunos problemas epistemológicos de la ciencia de la información a partir de elementos vertidos por Rorty, en Saraiva (2019).

${ }^{5}$ La obra de Richard Rorty es muy abundante, y ha sufrido diversas interpretaciones y discusiones.La presente investigación se centrará en algunas de las tesis expuestas en La filosofía y el espejo de la naturaleza (1995). Para consultar las varias críticas realizadas a las propuestas de Rorty, se puede consultar Fernández Membrive (2015), Kalpokas (2005), Navia (2008).

${ }^{6}$ El segundo artículo de la serie sobre los fundamentos de la ciencia de la información versa sobre la adecuación de las herramientas cuantitativas al trabajo de las ciencias sociales y de cómo las debe adoptar la ciencia de la información (Brookes, 1980b). En el tercer artículo Brookes centra su atención sobre la medición cuantitativa de la información (Brookes, 1980c). En su último artículo trata sobre el cambio de paradigma y los requerimientos necesarios para su acogimiento (Brookes, 1981). 